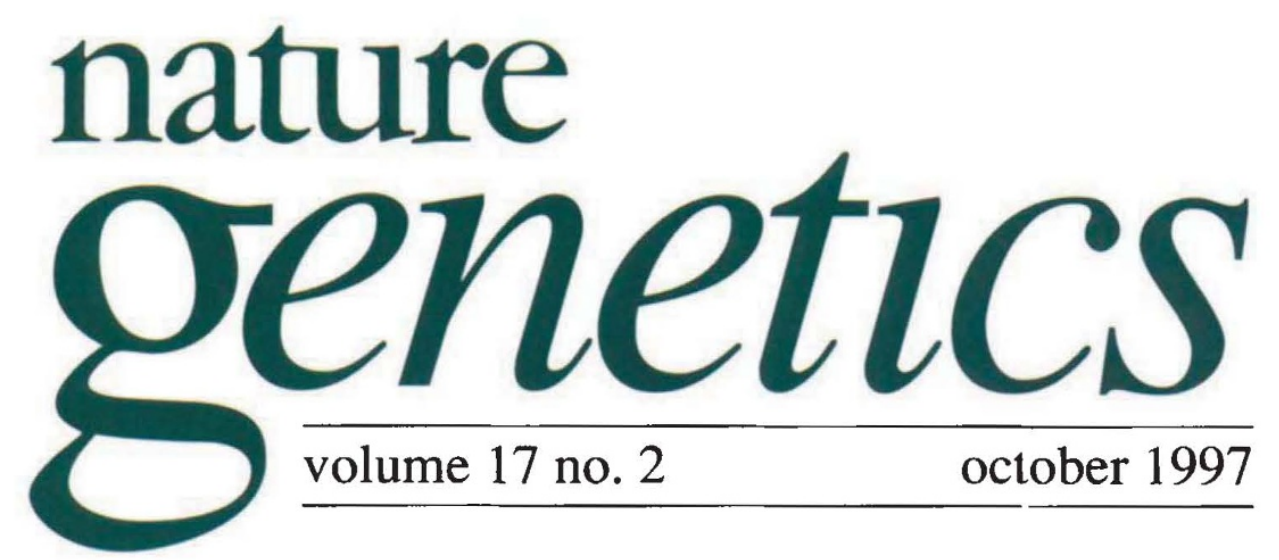

\title{
Come together
}

As did old flat-top in that memorable ballad by John Lennon, diabetes has been "grooving up" slowly, with respect to yielding clues to its genetic aetiology. A timely meeting* on the sanative shores of the Baltic sea, in Ystad, Sweden, at the end of July assessed recent progress in understanding the disease, with an eye to analytical approach. In his opening remarks, Leif Groop explained his rationale for choosing a Viking ship as a symbol for the meeting - "the field of diabetes research has been like the Viking wars, with pillage and plunder". He expressed the hope that by bringing all the major players together, consequent coordination may lead to more common efforts.

Arduous and painstaking in their execution, human genome scans for noninsulin dependent diabetes mellitus (NIDDM), have so far yielded only suggestive linkage and infrequent replication, while insulin-dependent diabetes mellitus (IDDM) lays claim to six 'confirmed' regions, IDDM1, 2, 4, 5, 8 and 10.

Leena Peltonen emphasized that NIDDM is not the only polygenic disorder refractory to genetic resolution; genome scans for multiple sclerosis in the Finnish population have also failed to deliver loci of significant linkage. Following a fortifying cup of coffee, a series of NIDDM genome scans were reviewed. Oluf Pederson described the design of an ongoing QTL analysis to determine loci that impact glucose tolerance and effectiveness and insulin secretion and sensitivity in Danes. In noting that the pill affects insulin sensitivity, he illustrated one of the challenges of NIDDM - the confounding effect of environmental influence.

Graeme Bell presented data on a population of Mexican Americans with a stag-

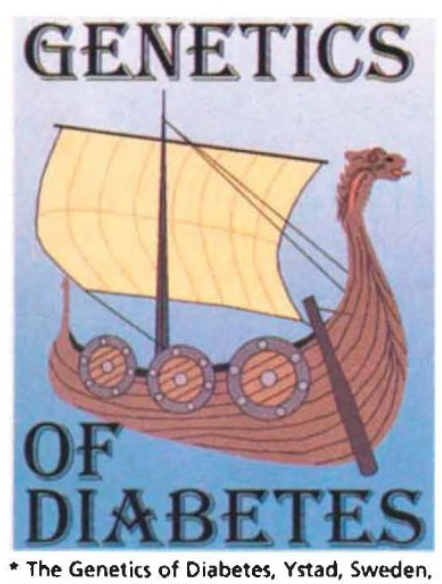
gering NIDDM rate of $50 \%$ in adults over the age of 35 , in which he last year mapped the 'NIDDMI' locus to chromosome 2 (other groups, however, have failed to replicate this finding). In answer to a question, he delivered the startling news that he could be "one gel away" from identifying the gene that underlies the locus; positional cloning of a polygene would certainly represent a landmatk in the genetic analysis of complex diseases, as underscored by Francis Collins. The extent to which such a gene may predispose towards diabetes in other populations remains to be determined, although it seems unlikely that many genes will have broad relevance to the corporate identity of NIDDM; lack of correspondence between scan peaks and the known MODY (mature-onset diabetes in the young) loci suggest that this is the case with the MODY genes, whose discoveries were reviewed by Philippe Froguel (for a strong tip on MODY4, see page 138).

Refinement of diagnostic parameters may result in obtaining linkage, as suggested by Melanie Mahtani, who presented results from a genome scan of affected individu- 
als from Botnia, Finland. No evidence for linkage was obtained when families were analysed together, but dividing the population according to insulin levels in those affected revealed evidence for linkage with a chromosome- 12 locus, although the linkage was not replicated in a separate collection. By a similar strategy, the FUSION (Finland-US Investigation of NIDDM genetics) group, represented by Soumitra Ghosh, has shown suggestive linkage on chromosome 20. Although suggestive linkage is, by definition, only suggestive, three other groups-led by Philippe Froguel, Andrzej Kroweleski and Don Bowden - have also found suggestive linkage for this locus, an encouraging sign that the locus is 'real' and that it is relevant to different populations, albeit those of European descent. Intriguingly, Clifton Bogardus reported linkage between this region and respiratory quotient in PIMA Indians. Efforts are underway to determine whether or not this locus is distinct from that of MODY1; preliminary findings suggest that it is. Provocatively, a peculiar type of double linkage was observed by Natalie Vionnet-on scanning the genome of a large French family, a single locus was linked by descent to late-onset NIDDM; in addition, this locus showed $100 \%$ concordance with the 'don't-reveal-the-chromosome' syndrome.

The genetic dissection of IDDM also provides testimony to the proverb: "there's no fun like work". Linda Wicker's presentation on mapping Idd loci by noting their effects in congenic strains of mice provided a demonstration of how rodents can be used to determine epistatic effect and to map the genes behind it. She reported that the finger of blame for $I d d 3$ now points to Il2, although the evidence is not yet conclusive. Other facets of IDDM biology were covered by John Todd, who speculated that IDDM2 may influence the level of pro-insulin peptide binding MHCII; this could explain the epistatic interaction between IDDM1 and IDDM2.

The genetics of human obesity, which has a well-documented alliance with NIDDM, is not as thoroughly charted. As reviewed by Jeff Friedman and Rudy Leibel, relevant genes have been established in animal models, their human counterparts identified, and their pathways explored. Some genome scans have been carried out, one of which implicates POMC on chromosome 2 in an Hispanic American population, as reported by Tony Comuzzie. Future efforts will benefit from the trials and tribulations of those who have been grappling with diabetes scans for a number of years.

The meeting underscored a perennial conflict-how can the quest for personal glory be reconciled with the need for the cooperation that permits progress? Individual efforts still dominate, and in keeping with the unfortunate and recent trend towards even greater secrecy in the sphere of human genetic research, many presented published results rather than new data. What will it take to move forward? Progress will come with time, better technology and approaches (such as those described by Mike Boehnke and Leonid Kruglyak) and unified efforts. The dissection of polygenic disorders is in ascent, and most would prefer to see the summit, rather than growing "old and grey" on the nursery slopes, waiting for progess with individual small samples - a potential scenario bemoaned by Todd.

Indeed, it is time to come together. Psychiatric genetics has shown that joint studies can be carried out-linkages reported by one group have been tested in collaborative effort between all the major players (importantly) including those without positive results in the region (see page 127). Perhaps a similar sense of desperation will drive those who study diabetes and obesity to the same end, a strategy worth trying for the patient, whose central role in the diabetes 'equation' was emphasized by Peter Goodfellow. It is, however, clear that personal glory, as manifested by firstauthor papers, is a critical component in advancing professionally and any attempt to encourage co-operation must address this primary obstacle. For the Vikings, the ship was a symbol of expansion. Hopefully, its use as a symbol is also a harbinger of future diabetes research. Eric Lander noted that the sail currently blows in opposite direction to the bow, as depicted in the logo; it is time to rerig the sail so that the ship may advance into new waters. 